\title{
Editorial
}

\section{Managing out-of-hospital cardiac arrest survivors: 2. Cardiological perspective}

In the first article in this series (Heart 2001;85:6-8) the initial assessment and management of unconscious out-ofhospital cardiac arrest survivors was discussed. Early management is centred around providing haemodynamic and ventilatory support, until it becomes apparent whether or not neurological recovery will occur. Thereafter the focus shifts towards identification of the cause of cardiac arrest (in patients in whom this was not evident at presentation). This is important because interventions such as revascularisation, antiarrhythmic drugs, and implantable cardioverter defibrillators (ICDs) significantly reduce the risk of subsequent death in specific patient subgroups. ${ }^{1-3}$

\section{Identification of the substrate of cardiac arrest} Approximately $40 \%$ of out-of-hospital cardiac arrest victims have the underlying substrate of acute myocardial infarction. ${ }^{4}$ The issue of whether or not to give thrombolysis to these patients if they have received prolonged cardiopulmonary resuscitation (CPR) is a difficult one. Trials provide conflicting information about the incidence of serious haemorrhagic complications..$^{5-8}$ Cited complications include haemothorax, and hepatic and retroperitoneal haemorrhage. The incidence of major bleeding is reportedly as high as $19 \%$, although most studies cite much lower figures. It is reasonable to consider thrombolysis if there is a clear history of chest discomfort preceding collapse, and there is no indication that the patient has suffered head trauma or a primary intracerebral haemorrhage. A computed tomographic head scan may be required to clarify this. Thrombolysis is contraindicated if significant thoracic trauma has occurred, so it is sensible to examine a chest $x$ ray before proceeding. As the benefits of thrombolysis may be partly offset by an increased risk of major haemorrhage after prolonged CPR, it should probably be reserved for patients with a widespread infarct territory (usually patients with anterior infarction), or infarction associated with haemodynamic compromise. ${ }^{8}$ If there is concern about the safety of thrombolysis, then primary angioplasty can be considered. Primary angioplasty and thrombolysis confer greatest benefit when given within eight hours of the onset of symptoms. Whether or not these interventions are used, aspirin should be given, if necessary intravenously or as a soluble preparation via a nasogastric tube. The only absolute contraindications are true aspirin allergy and active gastrointestinal bleeding.

In patients without clear electrocardiographic evidence of transmural myocardial infarction, the cardiac arrest usually relates to a pre-existing chronic cardiac condition. However, the possibility of an underlying non-cardiac cause should also be considered. This is especially true among patients who have suffered electromechanical dissociation, in whom treatable causes such as pulmonary embolism, drug overdosage or gastrointestinal haemorrhage may exist. Other reversible factors, such as electrolyte disturbance, proarrhythmic medication, systemic hypoxia, and sepsis should also be sought. If no obvious cause of cardiac arrest is found, a toxicology screen should be undertaken in case deliberate or accidental drug overdosage has occurred.

\section{Minimising risk: pre-discharge management}

After haemodynamic stabilisation and management of immediate complications, in many cases after recovery from a period of unconsciousness, patients' long-term risk of malignant arrhythmia has to be assessed.

ACUTE MYOCARDIAL INFARCTION

Patients who survive cardiac arrest which occurs within 48 hours of an acute myocardial infarction have a relatively good long term prognosis. Their subsequent risk of cardiac arrest is little higher than that of patients with uncomplicated myocardial infarction, and their management is no different. ${ }^{9}$ However, myocardial infarction may be difficult to diagnose because the history is absent or unclear, the ECG may not show a typical infarct pattern, and some biochemical markers are deranged by trauma to skeletal muscle. Cardiospecific markers such as creatine kinase MB mass are extremely helpful in this situation. ${ }^{10}$

CARDIAC ARREST WITHOUT MYOCARDIAL INFARCTION In patients who have not had an acute myocardial infarction, investigations are directed at identifying underlying ischaemic or structural heart disease. Most have ischaemic heart disease, often with a previous myocardial infarction and left ventricular dysfunction. The history may be helpful; an account of antecedent chest discomfort points towards an ischaemic aetiology. Cardiac arrest associated with exercise may be caused by myocardial ischaemia, or by catecholamine sensitive arrhythmias found in patients with the long QT syndrome (LQTS) and hypertrophic cardiomyopathy. ${ }^{11}{ }^{12}$ Exercise electrocardiography helps in their evaluation. Echocardiography helps identify structural substrates such as infarcted segments or left ventricular aneurysm, valve defects (for example, aortic stenosis, mitral valve prolapse), and cardiomyopathies. Coronary angiography is virtually mandatory before discharge if myocardial infarction is excluded, whether or not ischaemia is demonstrated by non-invasive testing, because revascularisation may significantly improve some patients' prognosis. ${ }^{1}$ It is sensible to reassess patients with electrophysiological testing after revascularisation, as it is otherwise difficult to know whether the substrate for ventricular arrhythmia has been removed. ${ }^{13}$

For cardiac arrest victims in whom a transient, reversible cause is not identified as the trigger, ICDs reduce the risk of arrhythmic death and appear to be superior to antiarrhythmic drug treatment. ${ }^{314}$ Many of these patients have ischaemic heart disease with left ventricular impairment caused by prior myocardial infarction. Patients with idiopathic dilated cardiomyopathy who survive cardiac arrest are also at high risk of sudden death. In the antiarrhythmics versus implantable defibrillator (AVID) trial, the subgroup with dilated cardiomyopathy appeared to derive the same benefit from ICD treatment as patients 
with ischaemic heart disease. ${ }^{3}$ It is more difficult to prove benefit in smaller subgroups, such as cardiac arrest victims with hypertrophic cardiomyopathy, LQTS, or adult congenital heart disease, but it is likely that these groups will also benefit. However, ICD treatment can be associated with significant psychological morbidity, because of inappropriate (or appropriate) defibrillator shocks. Adjunctive drug treatment, to reduce the incidence of arrhythmic episodes, was necessary in over one quarter of patients enrolled in the major ICD trials.

The role of programmed electrical stimulation (PES) is changing in the era of the ICD. Whereas previously PES was used to guide antiarrhythmic drug treatment, it is now recognised that empiric amiodarone treatment (ideally in conjunction with $\beta$ blockade), and ICD implantation confer equivalent or superior benefit in terms of mortality reduction. ${ }^{2}{ }^{14}$ The sensitivity of PES is also questionable in resuscitated cardiac arrest survivors without an identified, reversible cause. The American Heart Association currently recommends ICD implantation in all of these patients. Despite these limitations, PES can provide useful information about the nature of ventricular tachycardia (for example, rate, and response to overdrive pacing) that helps direct ICD programming. Additional electrophysiological testing is useful for evaluating patients resuscitated from cardiac arrest in whom the arrest rhythm is not documented. Here, sinoatrial (SA) and atrioventricular (AV) nodal dysfunction (which can precipitate bradycardic arrest and sometimes secondary ventricular arrhythmias) and occult accessory pathways (associated with pre-excited atrial fibrillation and ventricular fibrillation) may be identified and characterised.

CARDIAC ARREST WITH AN APPARENTLY NORMAL HEART A minority of out-of-hospital cardiac arrest victims exhibit no obvious evidence of structural or ischaemic heart disease using the above investigations. In these patients rarer diagnoses should be considered. Magnetic resonance imaging helps diagnose cardiac infiltration (for example, sarcoidosis, tumours) and arrhythmogenic right ventricular cardiomyopathy. LQTS may present with subtle patterns of QT interval prolongation, and this diagnosis should be considered in patients with a family history of syncope, "seizure disorder", or sudden death. In these patients $\beta$ blockade and/or stellectomy reduce the incidence of autonomically driven tachycardia, although ICD implantation should be strongly considered if cardiac arrest has occurred. The syndrome of right bundle branch block, ST segment elevation, and sudden death (Brugada syndrome) may also be occult; administration of a class I antiarrhythmic agent such as flecainide will unmask the ECG pattern in these cases.

WHEN IS ICD TREATMENT NOT INDICATED?

There are several situations in which ICD treatment is not indicated after out-of-hospital cardiac arrest. ICDs are not indicated for patients with cardiac arrest caused by evolving myocardial infarction, electrolyte abnormalities, and other transient, reversible causes. For patients with pre-excitation syndromes in which ventricular fibrillation has arisen as a result of pre-excited atrial fibrillation, radiofrequency ablation is the preferred treatment. (Ablation may also have an adjunctive role in ICD treated patients with ventricular tachycardia who suffer from frequent arrhythmias and recurrent device shocks). ICDs are contraindicated in patients with incessant or very frequent ventricular arrhythmias. In these cases catheter ablation, endocardial resection, or cardiac transplantation should be considered. Also, the survival benefit from ICD treatment is very limited in patients with severe, drug refractory cardiac failure that are not candidates for cardiac transplantation, and in patients with other terminal illnesses.

With increasing public awareness of basic life support skills, and the provision of rapid defibrillation by the emergency services, the number of individuals who survive outof-hospital cardiac arrest is set to increase. Identification of the cause of their cardiac arrest is central to risk assessment and management. Most have underlying ischaemic heart disease, towards which initial investigations are directed. The indications for ICD treatment are expanding rapidly, and this is the treatment of choice for most patients in whom myocardial infarction or other reversible or transient triggers have been excluded. Antiarrhythmic drugs continue to have a role in patients whose prognosis is limited by comorbidity, and as adjuvant therapy. ICD treatment is currently expensive, and in countries where budget limitations confine the use of ICDs to higher risk subgroups, antiarrhythmic drugs continue to be used as first line treatment for many patients.

University of Edinburgh Cardiovascular Unit,

NEIL R GRUBB

Cardiology Department

Royal Infirmary,

1 Lauriston Place,

Edinburgh EH3 9YW, UK

N.Grubb@ed.ac.uk.

1 Bigger JT, for the Coronary Artery Bypass Graft (CABG) Patch Trial Investigators. Prophylactic use of implanted cardiac defibrillators in patients at high risk for ventricular arrhythmias after coronary artery bypass graft surgery. $\mathrm{N} \mathrm{Engl} \mathrm{F} \mathrm{Med} \mathrm{1997;337:1569-75.}$

2 The CASCADE Investigators. Randomised antiarrhythmic drug therapy in survivors of cardiac arrest (the CASCADE study). Am $\mathcal{f}$ Cardiol 1993;72:280-7.

3 The Antiarrhythmics versus Implantable Defibrillator (AVID) Investigators. A comparison of antiarrhythmic drug therapy with implantable defibrillators in patients resuscitated from near-fatal ventricular arrhythmias. $N$ Engl tors in patients resuscitate

4 Cobbe SM, Redmond MJ, Watson JM, et al. "Heartstart Scotland"-initial experience of a national scheme for out of hospital defibrillation. BMF 1991;302:1517-20

5 Druwe PM, Cools J, De Raedt HJ, et al. Liver rupture after cardiopulmonary resuscitation in a patient receiving thrombolytic therapy. Resuscitation 1996;32:213-16

6 Tenaglia AN, Califf RM, Candela RJ, et al. Thrombolytic therapy in patients requiring cardiopulmonary resuscitation. Am f Cardiol 1991;68:1015-19.

7 Scholz KH, Tebbe U, Herrmann C, et al. Frequency of complications of cardiopulmonary resuscitation after thrombolysis during acute myocardial infarction. Am 7 Cardiol 1992:69:724-8.

8 The ISIS-2 Collaborative Group. Randomised trial of intravenous streptokinase, oral aspirin, both or neither among 17817 cases of suspected acute nase, oral aspirin, both or neither ans

9 Volpi A, Cavalli A, Grazia Franzosi M, et al and the GISSI Investigators. One year prognosis of primary ventricular fibrillation complicating acute myocardial infarction. Am f Cardiol 1989;63:1174-8.

10 Zabel M, Koster W, Hohnloser SH. Usefulness of CK-MB and troponin T determinations in patients with acute myocardial infarction complicated by ventricular fibrillation. Clin Cardiol 1993;16:23-5.

11 Moss AJ, Robinson J. Clinical features of the idiopathic long QT syndrome. Circulation 1992;85(suppl I):I-140-4

12 Gregoratos G, Cheitlin MD, Conill A, et al. ACC/AHA guidelines for implantation of cardiac pacemakers and antiarrhythmia devices. $\mathcal{F}$ Am Coll Cardiol 1998;31:1175-206.

13 Manolis AS, Rastegar H, Mark Estes NA. Effects of coronary artery bypass grafting on ventricular arrhythmias: Results with electophysiological testing and long-term follow-up. PACE 1993;16:984-91.

14 Moss AJ, Hall WJ, Cannom DS, et al. Improved survival with an implanted defibrillator in patients with coronary artery disease at high risk for ventricular arrhythmia. N Engl f Med 1996;335:1933-40. 